\title{
Article \\ Excitations of the $n S$ States of Atomic Hydrogen by Electron Impact, Excitation Rate Coefficients, and Phase Shifts: Comparison with Positron Impact Excitation
}

\author{
Anand K. Bhatia
}

check for

updates

Citation: Bhatia, A.K. Excitations of the $n S$ States of Atomic Hydrogen by Electron Impact, Excitation Rate

Coefficients, and Phase Shifts:

Comparison with Positron Impact

Excitation. Atoms 2022, 10, 5.

https://doi.org/10.3390/

atoms10010005

Academic Editor:

Jean-Christophe Pain

Received: 6 December 2021

Accepted: 27 December 2021

Published: 31 December 2021

Publisher's Note: MDPI stays neutral with regard to jurisdictional claims in published maps and institutional affiliations.

Copyright: (C) 2021 by the author. Licensee MDPI, Basel, Switzerland. This article is an open access article distributed under the terms and conditions of the Creative Commons Attribution (CC BY) license (https:/ / creativecommons.org/licenses/by/ $4.0 /)$.
Heliophysics Science Division, NASA/Goddard Space Flight Center, Greenbelt, MD 20771, USA; anand.k.bhatia@nasa.gov

\begin{abstract}
The excitation cross-sections of the $n S$ states of atomic hydrogen, $n=2$ to 6 , by electron impact on the ground state of atomic hydrogen were calculated using the variational polarizedorbital method at various incident electron energies in the range 10 to $122 \mathrm{eV}$. Converged excitation cross-sections were obtained using sixteen partial waves ( $L=0$ to 15). Excitation cross-sections to $2 S$ state, calculated earlier, were calculated at higher energies than before. Results obtained using the hybrid theory (variational polarized orbital method) are compared to those obtained using other approaches such as the Born-Oppenheimer, close-coupling, R-matrix, and complex-exterior scaling methods using only the spherical symmetric wave functions. Phase shifts and elastic cross-sections are given at various energies and angular momenta. Excitation rate coefficients were calculated at various electron temperatures, which are required for plasma diagnostics in solar and astrophysics to infer plasma parameters. Excitation cross-sections are compared with those obtained by positron impact excitation.
\end{abstract}

Keywords: electron impact excitation; phase shifts; elastic cross-sections; excitation rate coefficients

\section{Introduction}

Cecilia Payne, a student of Eddington, suggested that hydrogen is the most abundant element in the universe. The electron impact excitation of hydrogen to the $2 p$ level resulting in Lyman-alpha radiation in astrophysics is well known. There is a possibility of exciting other levels, such as 2 s excitation resulting in two-photon decay. Excitation by positron impact is also possible. It is known that positrons are produced inside the Sun when protons collide with protons, contributing to the opacity of the atmosphere of the Sun, in addition to electrons. Their presence is indicated in the Sun as well as in the interstellar space by the detection of the $0.511 \mathrm{MeV}$ line from the center of the galaxy [1]. In earlier publications [2,3], cross-sections for excitation of the atomic hydrogen by electrons and positrons were presented. Cross-sections for excitation are a two-channel problem. They must include the initial state as well as the final state. Burke et al. [4], using the closecoupling approximation, carried out such a calculation. Their wave function includes both $1 S$ and $2 S$ states for $2 S$ excitation. Rudge [5] calculated excitation to the $2 S$ state at 3 incident energies using the Born-Oppenheimer approximation and compared his results with those obtained using the close-coupling approximation [4]. Bartlett and Stelbovics [6] used the Temkin-Poet models to study electron-hydrogen scattering and calculated excitation cross-sections to various $S$ states. Jones and Stelbovics [7] calculated excitation to $S$ states numerically using the Temkin-Poet model. Callaway [8] carried out calculations using 11-state expansion including 7 pseudostates. Scott et al. [9] carried out calculations using the $R$-matrix close-coupling approximation. There are other calculations in which excitation has been treated as a single-channel problem and where the initial wave function is considered to be exact. The earlier calculations [2] are now extended to excitation of the higher $S$ states of the hydrogen atom. Since the hydrogenic functions are 
exact, different approximations can be used to judge the possibility of obtaining accurate results. The present calculation was carried out using the variational polarized orbital method of Bhatia [10]. The asymptotic polarizability obtained in this approximation is $4.5 a_{0}^{3}$, the exact value. Even though the calculations were carried out as a single-channel approximation, the phase shifts obtained have lower bounds to the exact phase shifts and agree well with those obtained using other approximations. This method has been applied to calculate parameters of Feshbach resonances [11] and photoabsorption crosssections [12]. The results obtained are accurate, compare well with results obtained in other calculations, and agree with the experimental results. It seems justified to assume that the scattering functions required in the calculation of excitation cross-sections are accurate. Calhoun et al. [13], using the distorted-wave approximation, calculated excitation to $2 S$ state at 4 incident energies. Excitation to $2 S$ state was reported earlier [2]. It is expected that excitation cross-sections obtained using the present approach will be accurate for $n S$ states, for $n$ greater than 2 as well. In electron impact excitation experiments, the sum of excitation cross-sections and elastic scattering cross-sections might be measured. The present investigation would be useful then. Rydberg units are used throughout (electron mass $m_{e}=0.5, e^{2}=2, \hbar=1$ ). Distances are measured using Bohr radius $=1$, energies are in Rydberg $=13.605 \mathrm{eV}$, and $\mathrm{a}(-\mathrm{b})$ represents $\mathrm{a} \times 10^{(-\mathrm{b})}$.

\section{Theory and Calculations}

The method for calculating excitation cross-sections is discussed in detail in Ref. [2] and it is only briefly described here.

The excitation cross section from state ' $I$ ' to ' $f$ ' state is given by

$$
\sigma_{f i}=\frac{k_{f}}{k_{i}} \int\left|T_{f i}\right|^{2} d \Omega
$$

In the above equation, $k_{i}$ and $k_{f}$ are the initial and final momenta and the transition matrix $T_{f i}$ is given by

$$
T_{f i}=-\left(\frac{1}{4 \pi}\right)\left\langle\Psi_{f}|V| \Psi_{i}\right\rangle,
$$

The potential involved in the transition is given by

$$
V=-\frac{2 Z}{r_{1}}+\frac{2}{r_{12}}
$$

In the above expression, $r_{1}$ is the distance of the incident electron from the nucleus, assumed to be infinitely heavy, $r_{12}$ is the inter-electron distance, and $Z$ is the nuclear charge. In principle, the initial state wave is supposed to be an exact solution of the Schrödinger equation. It is given by

$$
\Psi_{i}\left(\vec{r}_{1}, \vec{r}_{2}\right)=\frac{1}{\sqrt{2}}\left[u\left(\vec{r}_{1}\right) \Phi^{p o l}\left(\vec{r}_{1}, \vec{r}_{2}\right) \pm(1 \leftrightarrow 2)\right]
$$

where $u\left(\vec{r}_{1}\right)$ is the scattering function and $\Phi^{p o l}\left(\vec{r}_{1}, \vec{r}_{2}\right)$ is the target wave function polarized due to the incident electron which produces a change in the energy equal to $-\frac{1}{2} \alpha E^{2}$, where $\alpha$ is the polarizability and $E$ is the electric field produced by the incident electron. Temkin and Lamkin [14] showed that in the presence of the incident electron the target wave function can be written as

$$
\Phi^{p o l}\left(\vec{r}_{1}, \vec{r}_{2}\right)=\phi_{0}\left(\vec{r}_{2}\right)-\frac{\chi_{S T}\left(r_{1}\right)}{r_{1}^{2}} \frac{u_{1 s \rightarrow p}\left(r_{2}\right)}{r_{2}} \frac{\cos \left(\theta_{12}\right)}{\sqrt{Z \pi}} .
$$


$\chi_{S t}$ is a smooth cutoff function introduced by Shertzer and Temkin [15], it is given by

$$
\chi_{S T}\left(r_{1}\right)=1-e^{-2 Z r_{1}}\left(\frac{\left(Z r_{1}\right)^{4}}{3}+\frac{4\left(Z r_{1}\right)^{3}}{3}+2\left(Z r_{1}\right)^{2}+2 Z r_{1}+1\right) .
$$

The function $u_{1 s \rightarrow p}\left(r_{2}\right)$ in Equation (5) is given by

$$
u_{1 s \rightarrow p}\left(r_{2}\right)=\left(\frac{Z}{2} r_{2}^{3}+r_{2}^{2}\right) e^{-Z r_{2}} .
$$

The unperturbed target function in the initial state is given by

$$
\phi_{0}\left(\vec{r}_{2}\right)=\sqrt{\frac{Z^{3}}{\pi}} e^{-Z r_{2}}
$$

The scattering function in the plane-wave normalization is given by

$$
u\left(\vec{r}_{1}\right)=\sqrt{4 \pi(2 L+1)} \frac{u\left(r_{1}\right)}{r_{1}} Y_{L 0}\left(\theta_{1}, \phi_{1}\right) .
$$

The spherical harmonic, $Y_{L 0}$, is a function of the angular momentum $L$ depending on spherical polar angles $\theta_{1}$ and $\phi_{1}$. The function $u\left(r_{1}\right)$ satisfies the Schrödinger equation:

$$
\int d \Omega_{1} d \vec{r}_{2} Y_{L 0}\left(\Omega_{1}\right) \Phi^{p o l}|H-E| \Psi_{i}\left(\vec{r}_{1}, \vec{r}_{2}\right)=0
$$

In the above equation, $E=E_{\text {target }}+k^{2}$ is the total energy, where $k^{2} \equiv k_{i}^{2}$ is the incident energy. $H$ is the Hamiltonian given by

$$
H=-\nabla_{1}^{2}-\nabla_{2}^{2}-\frac{2 Z}{r_{1}}-\frac{2 Z}{r_{2}}+\frac{2}{r_{12}}
$$

After integrating over $d \vec{r}_{2}$ and angles $\theta_{1}$ and $\phi_{1}$, the resulting integro-differential equation, which is quite complicated, is solved by a non-iterative method [16]. The method being used determine the initial state wave function in the present calculation is the variational polarized orbitals method [10], in which the phase shifts for scattering have lower variational bounds. The final wave function is given by

$$
\Psi_{f}\left(\vec{r}_{1}, \vec{r}_{2}\right)=e^{i \vec{k}_{f} \cdot \vec{r}_{1}} \phi_{n s}\left(\vec{r}_{2}\right) \text {. }
$$

The excited state target functions are $\phi_{n s}\left(\vec{r}_{2}\right), n=2$ to 6 . These functions $\phi_{n s}(\vec{r})$ are given in Appendix $\mathrm{A}$, and $k_{\mathrm{f}}$ is the final electron momentum after excitation.

\section{Results}

The excitation cross-sections were calculated at the incident momenta $k=0.87$ to 3.0 using 16 partial waves ( $\mathrm{L}=0$ to 15$)$. Convergence with the partial waves is shown in Table 2 of reference [2]. The $2 S$ excitation cross sections converge by $L=6$ or 7 , excitations to the higher $S$ states require more partial waves to get converged cross-sections.

Cross-sections for $1 S$ excitation to $2 S$, having 2 peaks, are shown again in Figure 1. It was indicated in reference [2] that the absolute value of $2 S$ excitation measured by Kauppila [17] is $0.163 \pm 0.020 \pi a_{0}^{2}$ at $11 \pm 0.2 \mathrm{eV}$, while the present calculation gives $0.137 \pi a_{0}^{2}$, in spite of including contributions of the higher partial waves (up to $L_{\max }=15$ ).

The excitation cross sections for $2 S, 3 S, 4 S, 5 S$, and $6 S$ are shown in Figures $1-5$. These figures are for $L_{\max }=12$ by which cross-sections have essentially converged. The excitation cross-sections $1 S$ to $3 S$ state have a peak at $k=1.1$, excitation to $4 S$ state at $k=1.02$, excitation to $5 S$ state at $k=1.05$, and excitation to $6 S$ state at $k=1.05$. The excitation cross-sections to $6 S$ state have another peak at $k=1.70$. This peak arises because for $L=0$, the cross-section 
is maximum at $k=1.70$. This fact has been verified by calculating cross-sections below and above $k=1.70$, but close to this $k$.

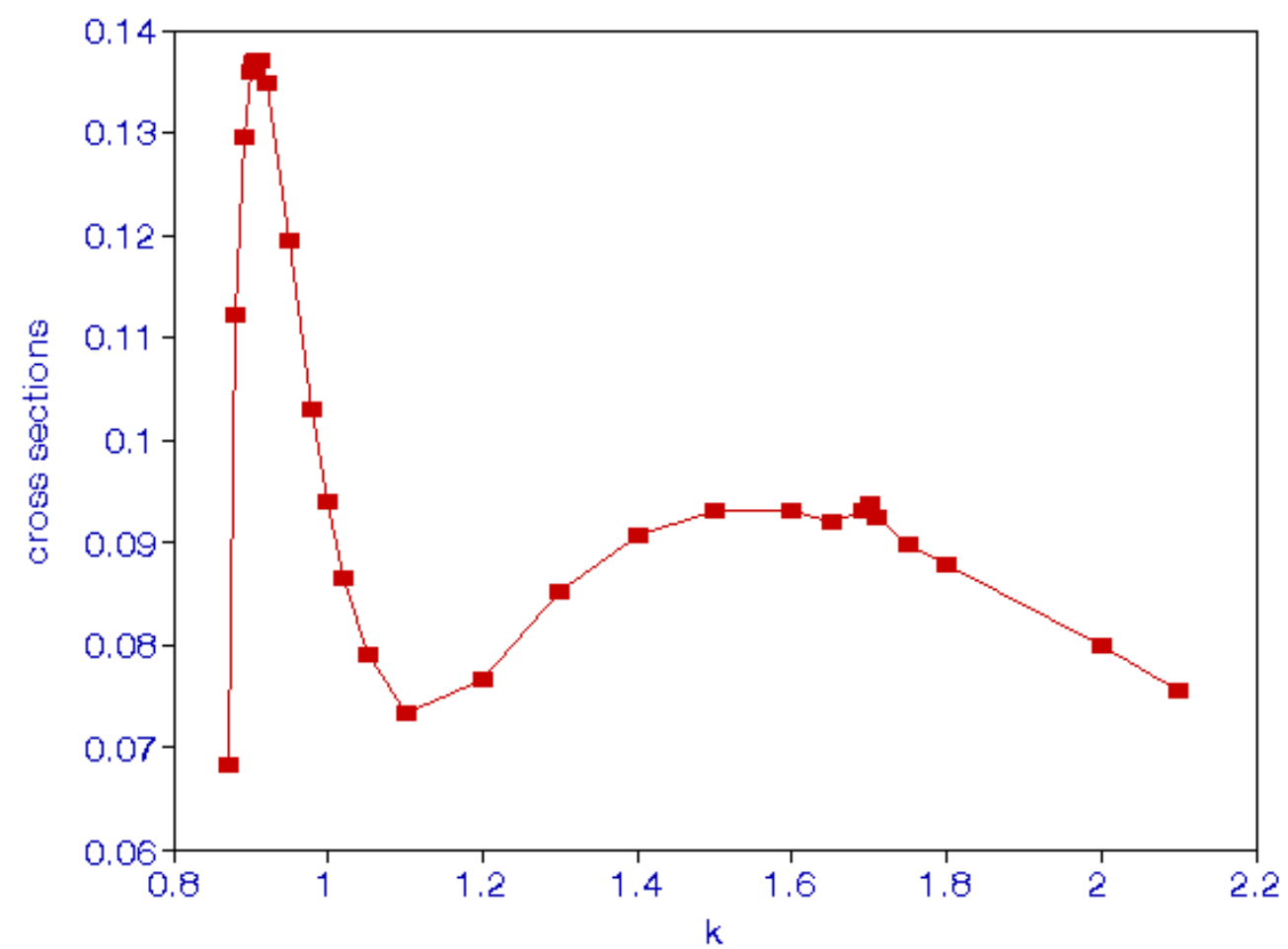

Figure 1. (Color online) Total $1 S$ to $2 S$ excitation cross sections $\left(\pi a_{0}^{2}\right)$ vs. the incident momentum $k$ (Ryd).

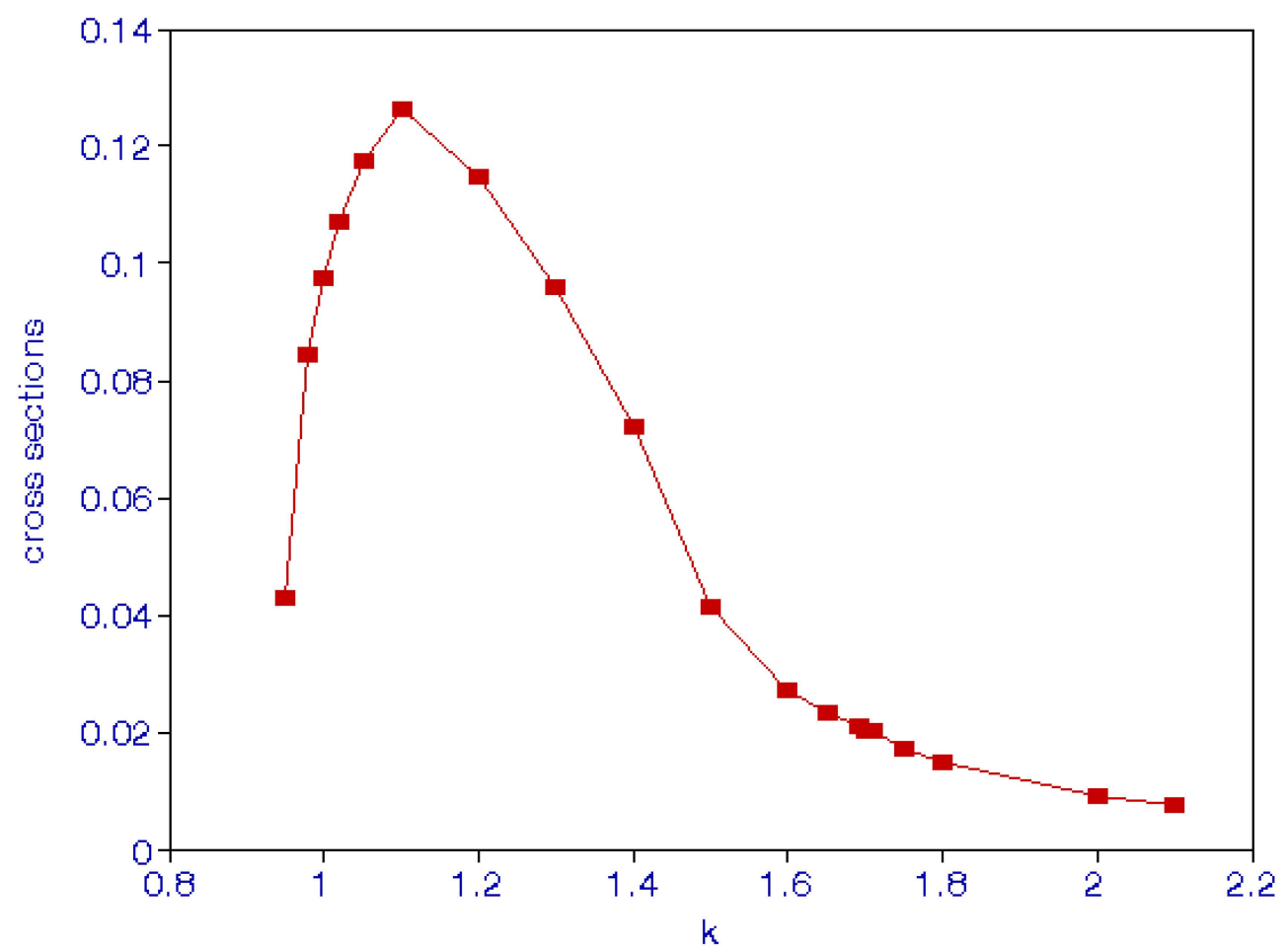

Figure 2. (Color online) Total $1 S$ to $3 S$ excitation cross sections $\left(\pi a_{0}^{2}\right)$ vs. the incident momentum $k$ (Ryd). 


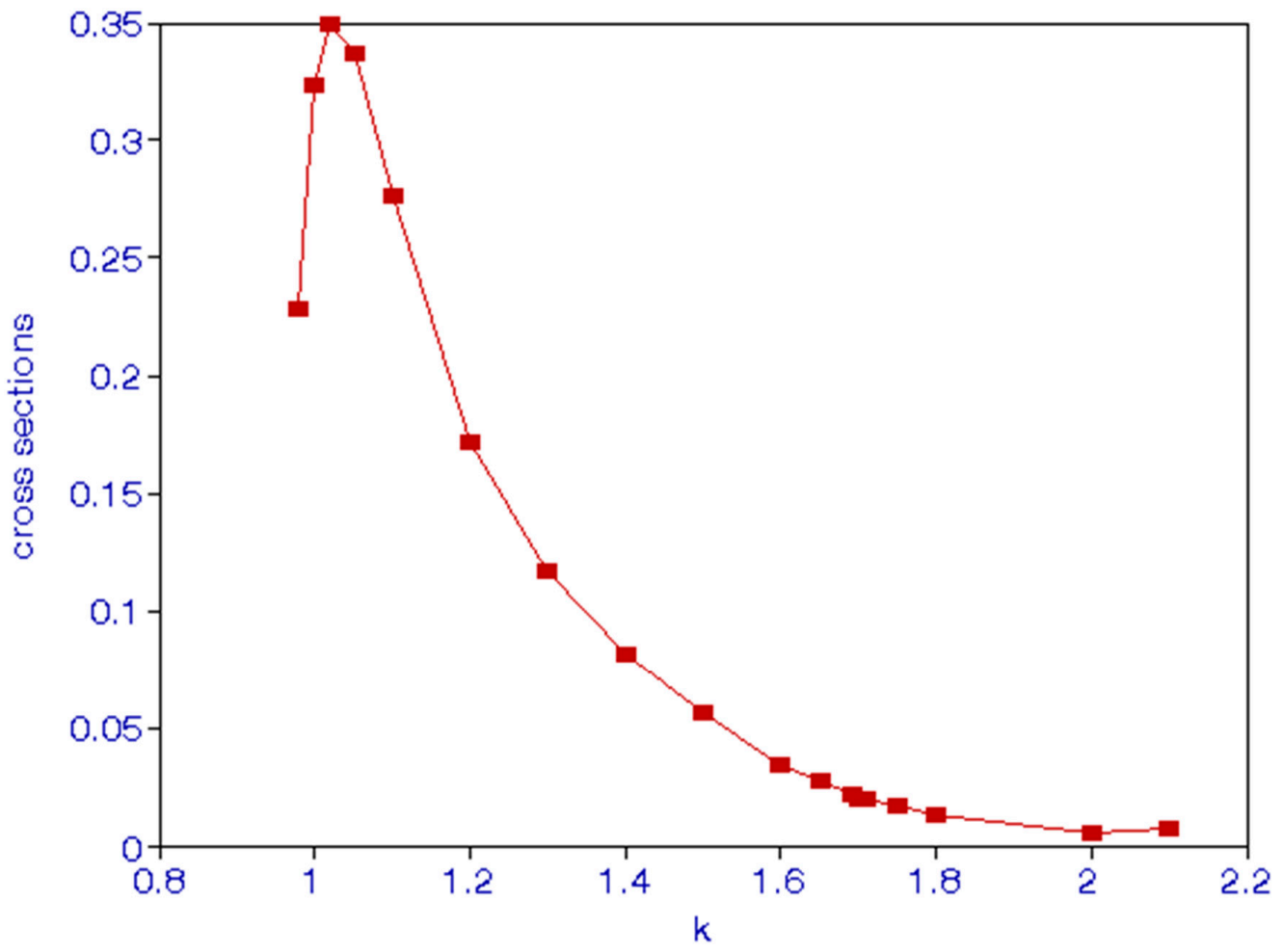

Figure 3. (Color online) Total $1 S$ to $4 S$ excitation cross sections $\left(\pi a_{0}^{2}\right)$ vs. the incident momentum $k$ (Ryd).

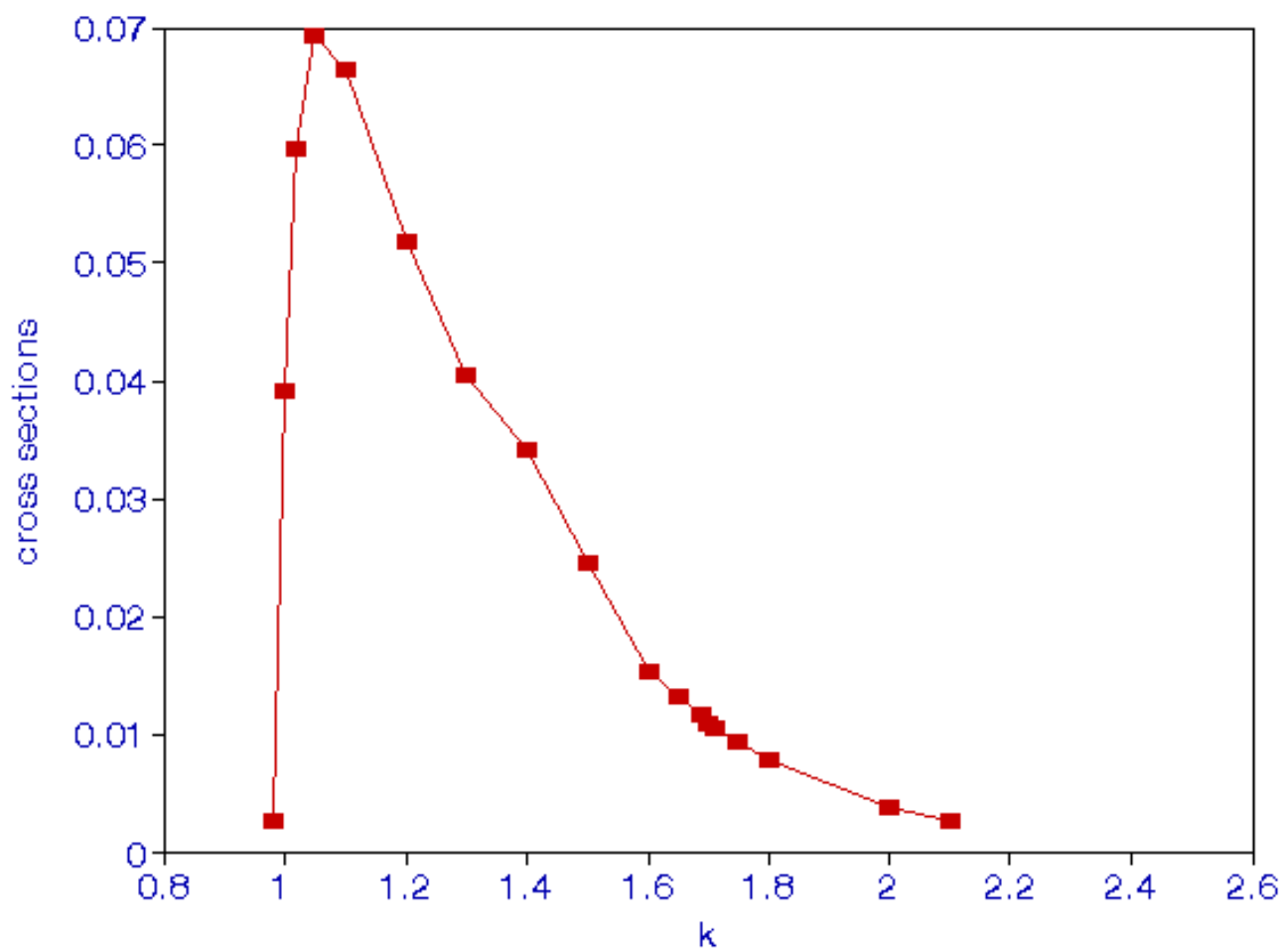

Figure 4. (Color online) Total $1 S$ to $5 S$ excitation cross sections $\left(\pi a_{0}^{2}\right)$ vs. the incident momentum $k$ (Ryd). 


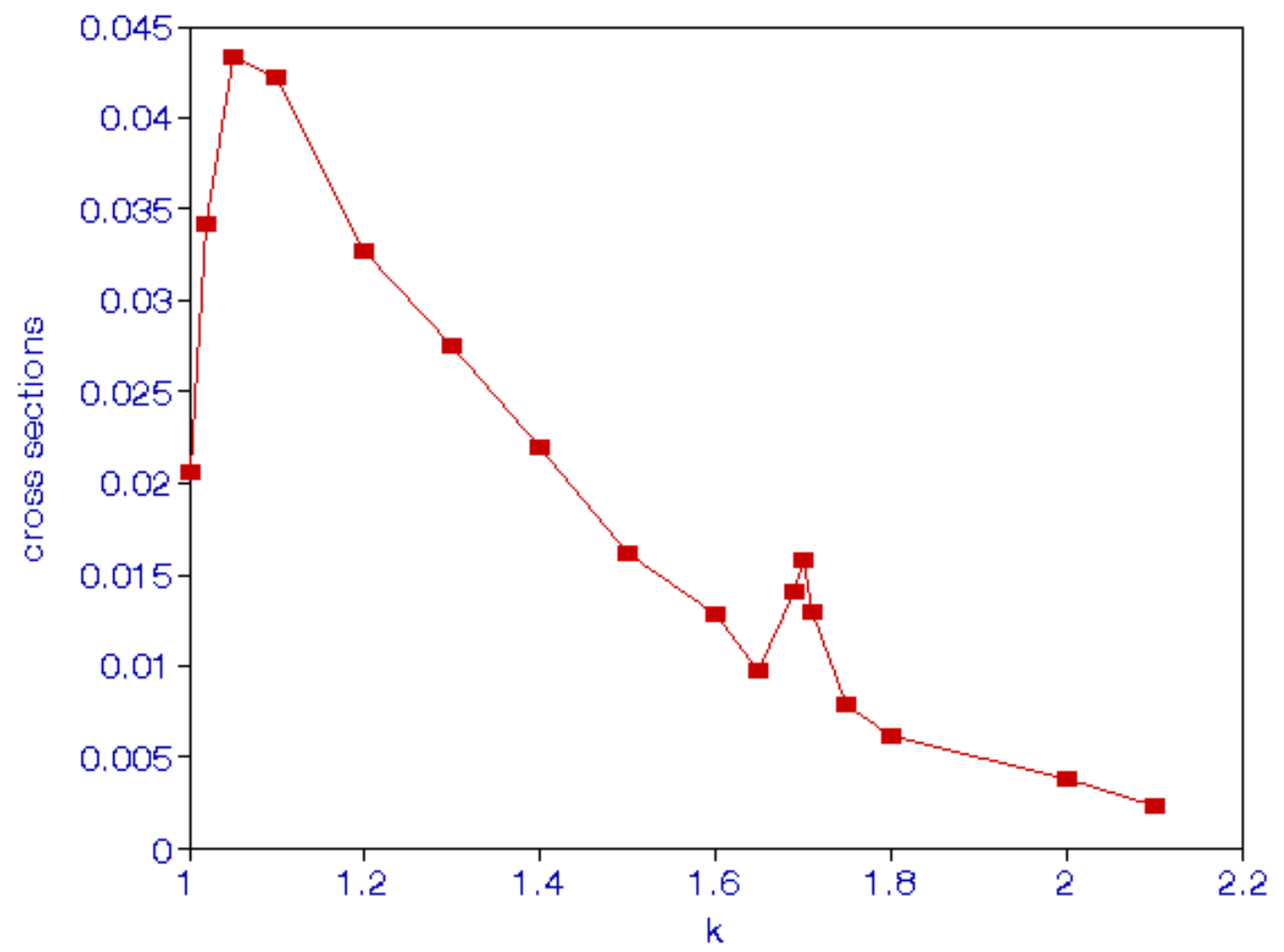

Figure 5. (Color online) Total $1 S$ to $6 S$ excitation cross sections $\left(\pi a_{0}^{2}\right)$ vs. the incident momentum $k$ (Ryd).

The excitation cross-sections obtained in the hybrid theory [10] are given in Table 1. The empty spaces indicate that the incident energies are not sufficient to excite these states. There was a typo in a routine in the code for calculating excitation cross-sections from $1 S$ state to $2 S$ state, reported in ref. [2]. That correction now has been made. The presently calculated excitations to the $2 S$ state differ in the 4 th or 5 th decimal place compared to those given in reference [2]. The excitation cross-sections to the $2 S$ state were compared to the results of the previous calculations (close-coupling, $R$-matrix, etc.) and those comparisons are not repeated here again.

The excitation cross-sections to the $3 S$ state have been calculated using the closecoupling [18] and the Born approximations by Whelan et al. [19]. Their results are higher than the present results. There are measurements of $3 S$ excitations by Mahan et al. [20]. Their results include cascades from higher states. Whelan et al. [19] indicate $0.013 \pi a_{0}^{2}$ for the cross section at $k=2.0$, which is lower than the result of Mahan et al. [20], which includes cascades from higher states. By $L_{\max }=15$, cross-sections have converged to at least to the 4th decimal place.

At $k=2.1$, the cross-section of the $6 S$ is $6.76(-5) \pi a_{0}^{2}$, and $k=2.6$, it is $8.33(-4)$ for $L=15$. Cross-sections start increasing at $k=2.5$. The sum of all these cross sections at each energy is also given in Table 1 , including elastic cross-sections. The present results should be accurate because the calculations were carried out using the quadruple precision. Callaway et al. [18] used six-state close-coupling expansion including an optical potential to calculate excitation to $2 S$ and $3 S$ states. The $2 S$ excitation results were already compared to those in [18] and $3 S$ excitations are now compared to those in [18] in Table 2. Bartlett et al. [21] carried out measurements at $12.24 \mathrm{eV}$ and compared their results for the excitation of the $3 S, 4 S$, and $5 S$ states with the results of exterior complex scaling calculations, the experimental uncertainty being 35\%. Their interpolated results for $3 S$ state are compared with the present results in Table 2 . Their results are lower compared to the present results. Similarly, their $4 S$ results are found to be lower than the present results. 
Table 1. Cross sections $\left(\pi a_{0}^{2}\right)$ of $\mathrm{n} S$ states of atomic hydrogen using the variational polarized orbital method with maximum $L_{\max }=15$.

\begin{tabular}{|c|c|c|c|c|c|c|c|c|}
\hline$k$ & $2 S$ & $3 S$ & $4 S$ & $5 S$ & $6 S$ & Total $^{\mathrm{a}}$ & $\begin{array}{c}\text { Elastic Cross } \\
\text { Section }\end{array}$ & Total1 ${ }^{b}$ \\
\hline 0.87 & $6.815(-2)$ & & & & & $6.815(-2)$ & 5.974 & 6.042 \\
\hline 0.88 & $1.123(-1)$ & & & & & $1.123(-1)$ & 5.899 & 6.011 \\
\hline 0.89 & $1.295(-1)$ & & & & & $1.295(-1)$ & 5.820 & 5.950 \\
\hline 0.90 & $1.359(-1)$ & & & & & $1.359(-1)$ & 5.538 & 5.674 \\
\hline 0.904 & $1.368(-1)$ & & & & & $1.368(-1)$ & 5.473 & 5.610 \\
\hline 0.905 & $1.369(-1)$ & & & & & $1.369(-1)$ & 5.456 & 5.593 \\
\hline 0.906 & $1.363(-1)$ & & & & & $1.363(-1)$ & 5.444 & 5.800 \\
\hline 0.907 & $1.369(-1)$ & & & & & $1.369(-1)$ & 5.428 & 5.565 \\
\hline 0.91 & $1.370(-1)$ & & & & & $1.370(-1)$ & 5.388 & 5.525 \\
\hline 0.92 & $1.349(-1)$ & & & & & $1.349(-1)$ & 5.266 & 5.401 \\
\hline 0.95 & $1.194(-1)$ & $4.299(-2)$ & & & & $1.624(-1)$ & 5.073 & 5.235 \\
\hline 0.98 & $1.030(-1)$ & $8.428(-2)$ & $9.456(-2)$ & $2.691(-2)$ & & $2.805(-1)$ & 5.282 & 5.566 \\
\hline 1.00 & $9.389(-2)$ & $9.765(-2)$ & $1.488(-1)$ & $3.929(-2)$ & $2.056(-2)$ & $4.002(-1)$ & 5.158 & 5.578 \\
\hline 1.02 & $8.653(-2)$ & $1.074(-1)$ & $1.716(-1)$ & $5.961(-2)$ & $3.412(-2)$ & $6.450(-1)$ & 5.022 & 5.473 \\
\hline 1.05 & $7.898(-2)$ & $1.187(-1)$ & $1.769(-1)$ & $6.936(-2)$ & $4.329(-2)$ & $6.487(-1)$ & 4.788 & 5.275 \\
\hline 1.10 & $7.345(-2)$ & $1.321(-1)$ & $1.582(-1)$ & $6.636(-2)$ & $4.222(-2)$ & $4.723(-1)$ & 3.753 & 4.225 \\
\hline 1.20 & $7.667(-2)$ & $2.489(-1)$ & $1.159(-1)$ & $5.276(-2)$ & $3.321(-2)$ & $5.275(-1)$ & 2.880 & 3.407 \\
\hline 1.30 & $8.518(-2)$ & $1.259(-1)$ & $8.863(-2)$ & $4.360(-2)$ & $2.771(-2)$ & $3.711(-1)$ & 2.532 & 2.903 \\
\hline 1.40 & $9.062(-2)$ & $1.012(-1)$ & $6.684(-2)$ & $3.594(-2)$ & $2.307(-2)$ & $3.177(-1)$ & 2.267 & 2.585 \\
\hline 1.50 & $9.327(-2)$ & $6.610(-2)$ & $4.768(-2)$ & $2.728(-2)$ & $1.787(-2)$ & $2.321(-1)$ & 2.046 & 2.298 \\
\hline 1.60 & $9.314(-2)$ & $4.206(-2)$ & $3.192(-2)$ & $1.808(-2)$ & $1.847(-2)$ & $2.037(-1)$ & 1.887 & 2.098 \\
\hline 1.65 & $9.216(-2)$ & $3.216(-2)$ & $2.688(-2)$ & $2.116(-2)$ & $1.134(-2)$ & $1.837(-1)$ & 1.888 & 2.071 \\
\hline 1.69 & $9.329(-2)$ & $2.683(-2)$ & $2.206(-2)$ & $1.400(-2)$ & $1.547(-2)$ & $1.716(-1)$ & 1.749 & 1.920 \\
\hline 1.70 & $9.377(-2)$ & $2.644(-2)$ & $2.012(-2)$ & $1.313(-2)$ & $1.715(-2)$ & $1.706(-1)$ & 1.116 & 1.287 \\
\hline 1.71 & $9.243(-2)$ & $2.565(-2)$ & $2.003(-2)$ & $1.261(-2)$ & $1.434(-2)$ & $1.651(-1)$ & 0.665 & 0.830 \\
\hline 1.75 & $8.987(-2)$ & $2.075(-2)$ & $1.712(-2)$ & $1.572(-2)$ & $8.288(-3)$ & $2.417(-1)$ & 1.060 & 1.302 \\
\hline 1.80 & $8.794(-2)$ & $1.742(-2)$ & $1.320(-2)$ & $8.702(-3)$ & $6.475(-3)$ & $1.338(-1)$ & 1.141 & 1.275 \\
\hline 2.00 & $8.002(-2)$ & $9.284(-3)$ & $5.747(-3)$ & $4.049(-3)$ & $3.424(-3)$ & $1.025(-1)$ & 0.926 & 1.029 \\
\hline 2.10 & $7.586(-2)$ & $7.706(-3)$ & $8.031(-3)$ & $2.906(-3)$ & $2.422(-3)$ & $9.693(-2)$ & 0.695 & 0.792 \\
\hline 2.20 & $7.177(-2)$ & $6.640(-3)$ & $5.778(-3)$ & $2.161(-3)$ & $1.896(-3)$ & $8.824(-2)$ & 0.634 & 0.722 \\
\hline 2.30 & $6.783(-2)$ & $5.861(-3)$ & $6.081(-3)$ & $1.689(-3)$ & $1.564(-3)$ & $8.303(-2)$ & 0.578 & 0.661 \\
\hline 2.40 & $6.406(-2)$ & $5.184(-3)$ & $1.732(-3)$ & $1.431(-3)$ & $1.343(-3)$ & $7.375(-2)$ & 0.528 & 0.602 \\
\hline 2.50 & $6.050(-2)$ & $5.680(-3)$ & $1.852(-3)$ & $1.759(-3)$ & $1.437(-3)$ & $7.122(-2)$ & 0.484 & 0.555 \\
\hline 2.60 & $5.711(-2)$ & $4.435(-3)$ & $2.808(-3)$ & $2.207(-3)$ & $1.847(-3)$ & $6.803(-2)$ & 0.445 & 0.514 \\
\hline 3.00 & $5.058(-2)$ & $5.804(-3)$ & $1.750(-2)$ & $6.023(-2)$ & $3.427(-3)$ & $1.375(-1)$ & 0.328 & 0.466 \\
\hline 3.20 & $4.081(-2)$ & $4.534(-3)$ & $1.057(-2)$ & $5.114(-3)$ & $3.111(-3)$ & $6.414(-2)$ & 0.286 & 0.350 \\
\hline
\end{tabular}

a Total: represents the sum of all the excitation cross sections. ${ }^{b}$ Total1: represents the sum of excitation cross sections and the elastic cross sections.

Table 2. Comparison of the present $3 S$ excitation cross sections $\left(\pi a_{0}^{2}\right)$ with those in references $[18,21]$.

\begin{tabular}{cccc}
\hline $\boldsymbol{k}$ & Present & Ref. [18] & Ref. [21] \\
\hline 0.95 & 0.043 & 0.036 & \\
1.00 & 0.09765 & & 0.000904 \\
1.10 & 0.1321 & & 0.001936 \\
1.20 & 0.250 & 0.039 & 0.002512 \\
1.30 & 0.1259 & & 0.002713 \\
1.40 & 0.101 & 0.0179 & 0.002613 \\
1.50 & 0.061 & 0.0146 & 0.002315 \\
1.60 & 0.042 & 0.0123 & 0.001879 \\
1.70 & 0.02644 & & 0.001395 \\
1.80 & 0.01792 & & 0.000949 \\
2.00 & 0.009284 & 0.0086 & 0.000467 \\
2.35 & 0.005523 & 0.0072 & \\
\hline
\end{tabular}


The converged close-coupling calculations of Bray et al. [22] gave for $2 S$ cross-sections equal to $0.252 a_{o}^{2}, 0.212 a_{0}^{2}$, and $0.139 a_{0}^{2}$ at $k$ equal to $1.60,2.0$, and 2.71 , respectively. Their results are lower than those given in Table 1 in units of $\pi a_{0}^{2}$. Calculations by Poet [23] using spherically symmetric wave functions and $R$-matrix calculations of Scott et al. [9] do not have excitation to $3 S$ state. We find that the results of the exterior complex scaling calculation [6] are lower while those obtained using the converged close-coupling calculations [22] are close to the present results. These approaches [6,18-22] are very complex compared to the present variational polarized orbital method, which is very easy to use and provides fairly accurate results.

Phase shifts at various incident energies are given in Table 3a-e for partial waves $L=0$ to 15 . They are variationally correct, that is, they are below the exact results. The triplet phase shifts are higher than the singlet phase shifts. In Table $3 b$, for $L=4$, at $k=2.6$ and $k=3.0$, phase shifts appear to be discontinuous. However, if $\pi$ radians are added, phase shifts are found to be continuous. These phase shifts have been used to calculate the elastic scattering cross-sections, which are also given in Table 1 along with the excitation cross-sections. The sum of the excitation cross-sections and elastic cross-sections would be useful when the measurements of total cross-sections are carried out.

Table 3. (a). Phase shifts (radians); (b). Phase shifts $\left(10^{-3}\right.$ radians); (c). Phase shifts $\left(10^{-3}\right.$ radians); (d). Phase shifts $\left(10^{-3}\right.$ radians); (e). Phase shifts $\left(10^{-3}\right.$ radians).

(a)

\begin{tabular}{|c|c|c|c|c|c|c|c|c|}
\hline \multirow[t]{2}{*}{$k$} & \multicolumn{2}{|c|}{$L=0$} & \multicolumn{2}{|c|}{$L=1$} & \multicolumn{2}{|c|}{$L=2$} & \multicolumn{2}{|c|}{$L=3$} \\
\hline & Triplet & Singlet & Triplet & Singlet & Triplet & Singlet & Triplet & Singlet \\
\hline 0.75 & 0.5837 & 0.5648 & & & & & & \\
\hline 0.87 & 1.5375 & 0.6624 & 0.3313 & 3.0692 & 0.0630 & 0.0339 & 0.0209 & 0.0212 \\
\hline 0.91 & 1.4909 & 0.6381 & 0.3343 & 3.0709 & 0.0667 & 0.0359 & 0.0221 & 0.0225 \\
\hline 0.92 & 1.4796 & 0.6326 & 0.3348 & 3.0714 & 0.0676 & 0.0365 & 0.0225 & 0.2283 \\
\hline 0.95 & 1.4467 & 0.6175 & 0.3361 & 3.0737 & 0.0702 & 0.0382 & 0.0235 & 0.0238 \\
\hline 0.98 & 1.4152 & 0.6043 & 0.3368 & 3.0766 & 0.0729 & 0.0399 & 0.0246 & 0.0248 \\
\hline 1.00 & 1.3949 & 0.9654 & 0.3371 & 3.0789 & 0.0746 & 0.0411 & 0.0252 & 0.0254 \\
\hline 1.02 & 1.3752 & 0.5875 & 0.3371 & 3.0814 & 0.0762 & 0.0423 & 0.0259 & 0.0260 \\
\hline 1.05 & 1.3468 & 0.5803 & 0.3369 & 3.0856 & 0.0787 & 0.0442 & 0.0270 & 0.0270 \\
\hline 1.10 & 1.3023 & 0.5682 & 0.3359 & 3.0937 & 0.0827 & 0.0474 & 0.0289 & 0.0286 \\
\hline 1.20 & 1.2236 & 0.5538 & 0.3326 & 3.1129 & 0.0903 & 0.0539 & 0.0327 & 0.0319 \\
\hline 1.30 & 1.1585 & 0.5492 & 0.3284 & 3.1347 & 0.0974 & 0.0604 & 0.0367 & 0.3515 \\
\hline 1.40 & 1.1083 & 0.5503 & 0.3241 & 0.0159 & 0.1040 & 0.0666 & 0.0410 & 0.0384 \\
\hline 1.50 & 1.0799 & 0.5426 & 0.3204 & 0.0387 & 0.1102 & 0.0723 & 0.0453 & 0.0417 \\
\hline 1.60 & 1.1128 & 0.5590 & 0.3172 & 0.6047 & 0.1159 & 0.0774 & 0.0496 & 0.0448 \\
\hline 1.65 & 1.2502 & 0.5612 & 0.3157 & 0.0708 & 0.1185 & 0.0798 & 0.0517 & 0.0464 \\
\hline 1.69 & 2.0018 & 0.5628 & 0.3145 & 0.0788 & 0.1206 & 0.0816 & 0.0634 & 0.0476 \\
\hline 1.70 & 2.5749 & 0.5631 & 0.3142 & 0.0808 & 0.1211 & 0.0820 & 0.0538 & 0.0479 \\
\hline 1.71 & 3.0948 & 0.5635 & 0.3140 & 0.0827 & 0.1216 & 0.0824 & 0.0542 & 0.0482 \\
\hline 1.75 & 1.1399 & 0.5648 & 0.3129 & 0.0903 & 0.1235 & 0.0841 & 0.0559 & 0.0494 \\
\hline 1.80 & 0.7113 & 0.5662 & 0.3117 & 0.0993 & 0.1258 & 0.0861 & 0.0579 & 0.0509 \\
\hline 2.00 & 0.7524 & 0.5681 & 0.3070 & 0.1312 & 0.1328 & 0.0930 & 0.0564 & 0.0655 \\
\hline 2.10 & 0.7391 & 0.5672 & 0.3048 & 0.1446 & 0.1370 & 0.0961 & 0.0588 & 0.0690 \\
\hline 2.20 & 0.7231 & 0.5652 & 0.3026 & 0.1565 & 0.1398 & 0.0990 & 0.0722 & 0.0612 \\
\hline 2.30 & 0.7067 & 0.5623 & 0.3004 & 0.1669 & 0.1422 & 0.1016 & 0.0751 & 0.0634 \\
\hline 2.40 & 0.6908 & 0.5586 & 0.2982 & 0.1760 & 0.1443 & 0.1042 & 0.0778 & 0.0655 \\
\hline 2.50 & 0.6756 & 0.5544 & 0.2959 & 0.1840 & 0.1460 & 0.1066 & 0.0802 & 0.0674 \\
\hline 2.60 & 0.6611 & 0.5498 & 0.2935 & 0.1909 & 0.1475 & 0.1089 & 0.0825 & 0.0692 \\
\hline 3.00 & 0.6102 & 0.5287 & 0.2841 & 0.2102 & 0.1516 & 0.1173 & 0.0898 & 0.0755 \\
\hline
\end{tabular}


Table 3. Cont.

\begin{tabular}{|c|c|c|c|c|c|c|}
\hline \multicolumn{7}{|c|}{ (b) } \\
\hline \multirow[t]{2}{*}{$k$} & \multicolumn{2}{|c|}{$\mathrm{L}=4$} & \multicolumn{2}{|c|}{$\mathrm{L}=5$} & \multicolumn{2}{|c|}{$\mathrm{L}=6$} \\
\hline & Triplet & Singlet & Triplet & Singlet & Triplet & Singlet \\
\hline 0.91 & 6.751 & 6.794 & 7.314 & 7.315 & 4.706 & 4.705 \\
\hline 0.92 & 6.732 & 6.775 & 7.439 & 7.440 & 4.793 & 4.792 \\
\hline 0.95 & 6.643 & 6.688 & 7.812 & 7.813 & 5.053 & 5.052 \\
\hline 0.98 & 6.514 & 6.559 & 8.186 & 8.187 & 5.317 & 5.315 \\
\hline 1.00 & 6.405 & 6.449 & 8.435 & 8.436 & 5.491 & 5.491 \\
\hline 1.02 & 6.281 & 6.323 & 8.685 & 8.685 & 5.670 & 5.491 \\
\hline 1.05 & 6.057 & 6.094 & 9.056 & 9.055 & 5.933 & 5.930 \\
\hline 1.10 & 5.608 & 5.632 & 9.675 & 9.672 & 6.375 & 6.371 \\
\hline 1.20 & 4.449 & 4.418 & 10.91 & 10.90 & 7.258 & 7.252 \\
\hline 1.30 & 3.017 & 2.889 & 12.15 & 12.12 & 8.139 & 8.127 \\
\hline 1.40 & 1.431 & 1.156 & 13.42 & 13.37 & 9.030 & 9.011 \\
\hline 1.50 & -0.226 & -0.701 & 14.74 & 14.64 & 9.930 & 9.901 \\
\hline 1.60 & -1.901 & -2.625 & 16.08 & 15.93 & 10.82 & 10.77 \\
\hline 1.65 & -2.717 & -3.583 & 16.77 & 16.58 & 11.26 & 11.21 \\
\hline 1.69 & -3.354 & -4.341 & 17.33 & 17.11 & 11.63 & 11.56 \\
\hline 1.70 & -3.510 & -4.529 & 17.47 & 17.24 & 11.72 & 11.65 \\
\hline 1.71 & -3.666 & -4.716 & 17.62 & 17.38 & 11.81 & 11.74 \\
\hline 1.75 & -4.274 & -5.455 & 18.19 & 17.92 & 12.18 & 12.10 \\
\hline 1.80 & -5.008 & -6.358 & 18.92 & 18.60 & 12.64 & 12.55 \\
\hline 2.00 & -7.533 & -9.630 & 21.99 & 21.42 & 14.58 & 14.41 \\
\hline 2.10 & -8.534 & -10.26 & 23.59 & 22.87 & 15.59 & 15.38 \\
\hline 2.20 & -9.354 & -12.25 & 25.21 & 24.34 & 16.65 & 16.38 \\
\hline 2.30 & -9.996 & -13.39 & 26.85 & 25.81 & 17.73 & 17.40 \\
\hline 2.40 & -10.46 & -14.14 & 28.49 & 27.27 & 18.85 & 18.46 \\
\hline 2.50 & -10.76 & -14.82 & 30.12 & 28.71 & 19.98 & 19.50 \\
\hline 2.60 & -10.89 & -15.31 & 31.73 & 30.13 & 21.14 & 20.57 \\
\hline 3.00 & 57.14 & 51.32 & 57.82 & 35.50 & 25.84 & 24.92 \\
\hline \multicolumn{7}{|c|}{ (c) } \\
\hline \multirow[t]{2}{*}{$k$} & \multicolumn{2}{|c|}{$\mathrm{L}=7$} & \multicolumn{2}{|c|}{$\mathrm{L}=8$} & \multicolumn{2}{|c|}{$\mathrm{L}=9$} \\
\hline & Triplet & Singlet & Triplet & Singlet & Triplet & Singlet \\
\hline 0.91 & 3.178 & 3.175 & 2.230 & 2.229 & 1.619 & 1.619 \\
\hline 0.92 & 3.237 & 3.237 & 2.275 & 2.275 & 1.653 & 1.653 \\
\hline 0.95 & 3.423 & 3.423 & 2.410 & 2.410 & 1.753 & 1.753 \\
\hline 0.98 & 3.612 & 3.612 & 2.548 & 2.548 & 1.857 & 1.857 \\
\hline 1.00 & 3.740 & 3.739 & 2.642 & 2.642 & 1.927 & 1.927 \\
\hline 1.02 & 3.868 & 4.868 & 2.737 & 2.737 & 1.998 & 1.998 \\
\hline 1.05 & 4.060 & 4.059 & 2.879 & 2.878 & 2.104 & 2.104 \\
\hline 1.10 & 4.384 & 4.383 & 3.119 & 3.119 & 2.286 & 2.286 \\
\hline 1.20 & 5.041 & 5.038 & 3.614 & 3.613 & 2.663 & 2.663 \\
\hline 1.30 & 5.702 & 5.697 & 4.120 & 4.118 & 3.053 & 3.052 \\
\hline 1.40 & 6.377 & 6.370 & 4.647 & 4.644 & 3.469 & 3.468 \\
\hline 1.50 & 7.058 & 7.047 & 5.184 & 5.180 & 3.900 & 3.898 \\
\hline 1.60 & 7.716 & 7.699 & 5.701 & 5.694 & 4.314 & 4.314 \\
\hline 1.65 & 8.043 & 8.024 & 5.958 & 5.950 & 4.521 & 4,518 \\
\hline 1.69 & 8.304 & 8.282 & 6.161 & 6.152 & 4.686 & 4.682 \\
\hline 1.70 & 8.370 & 8.347 & 6.213 & 6.203 & 4.727 & 4.724 \\
\hline 1.71 & 8.436 & 8.412 & 6.263 & 6.254 & 4.769 & 4.765 \\
\hline 1.75 & 8.699 & 8.671 & 6.468 & 6.456 & 4.934 & 4.929 \\
\hline 1.80 & 9.025 & 8.992 & 6.719 & 6.706 & 5.136 & 5.131 \\
\hline 2.00 & 10.36 & 10.30 & 7.730 & 7.707 & 5.948 & 5.938 \\
\hline 2.10 & 11.05 & 10.97 & 8.235 & 8.202 & 6.347 & 6.333 \\
\hline 2.20 & 11.76 & 11.66 & 8.747 & 8.708 & 6.744 & 6.727 \\
\hline
\end{tabular}


Table 3. Cont.

\begin{tabular}{lllllll}
\hline 2.30 & 12.49 & 12.37 & 9.267 & 9.219 & 7.144 & 7.123 \\
2.40 & 13.24 & 13.09 & 9.800 & 9.741 & 7.546 & 7.956 \\
2.50 & 14.02 & 13.84 & 10.35 & 1.0 .28 & 8.371 & 8.924 \\
2.60 & 14.82 & 14.61 & 10.91 & 10.83 & 10.23 & 10.15 \\
3.00 & 18.27 & 17.89 & 13.42 & 13.25 & & 10.23 \\
\hline
\end{tabular}

(d)

\begin{tabular}{|c|c|c|c|c|c|c|}
\hline \multirow[t]{2}{*}{$k$} & \multicolumn{2}{|c|}{$L=10$} & \multicolumn{2}{|c|}{$\mathrm{L}=11$} & \multicolumn{2}{|c|}{$\mathrm{L}=12$} \\
\hline & Triplet & Singlet & Triplet & Singlet & Triplet & Singlet \\
\hline 0.91 & 1.210 & 1.210 & 0.926 & 0.927 & 0.724 & 0.724 \\
\hline 0.92 & 1.236 & 1.236 & 0.947 & 0.947 & 1.404 & 1.404 \\
\hline 0.95 & 1.312 & 1.312 & 1.005 & 1.005 & 0.786 & 0.786 \\
\hline 0.98 & 1.390 & 1.390 & 1.066 & 1.066 & 0.834 & 0.834 \\
\hline 1.00 & 1.444 & 1.444 & 1.108 & 1.108 & 0.867 & 0.867 \\
\hline 1.02 & 1.498 & 1.498 & 1.150 & 1.150 & 0.900 & 0.900 \\
\hline 1.05 & 1.579 & 1.579 & 1.212 & 1.212 & 0.949 & 0.949 \\
\hline 1.10 & 1.718 & 1.718 & 1.320 & 1.320 & 1.034 & 1.034 \\
\hline 1.20 & 2.009 & 2.009 & 1.547 & 1.547 & 1.213 & 1.213 \\
\hline 1.30 & 2.314 & 2.134 & 1.787 & 1.787 & 1.494 & 1.404 \\
\hline 1.40 & 2.644 & 2.644 & 2.053 & 2.053 & 1.620 & 1.620 \\
\hline 1.50 & 2.992 & 2.992 & 2.992 & 2.992 & 1.854 & 1.854 \\
\hline 1.60 & 3.328 & 3.327 & 2.610 & 2.610 & 2.078 & 2.078 \\
\hline 1.65 & 3.497 & 3.495 & 3.497 & 3.495 & 2.191 & 2.191 \\
\hline 1.69 & 3.630 & 3.629 & 2.858 & 2.858 & 2.282 & 2.282 \\
\hline 1.70 & 3.665 & 3.664 & 3.665 & 3.664 & 2.306 & 2.305 \\
\hline 1.71 & 3.699 & 3.698 & 2.915 & 2.914 & 2.329 & 2.329 \\
\hline 1.75 & 3.835 & 3.832 & 3.834 & 3.832 & 2.422 & 2.422 \\
\hline 1.80 & 4.001 & 3.998 & 3.164 & 3.163 & 2.536 & 2.536 \\
\hline 2.00 & 4.672 & 4.667 & 3.725 & 3.723 & 3.007 & 3.006 \\
\hline 2.10 & 5.000 & 4.993 & 4.000 & 3.997 & 3,244 . & 3.239 \\
\hline 2.20 & 5.325 & 5.317 & 4.274 & 4.270 & 3.472 & 3.471 \\
\hline 2.30 & 5.649 & 5.639 & 4.545 & 4.540 & 3.703 & 3.701 \\
\hline 2.40 & 5.992 & 5.959 & 4.816 & 4.810 & 3.934 & 3.931 \\
\hline 2.50 & 6.297 & 6.282 & 5.085 & 5.078 & 4.162 & 4.159 \\
\hline 2.60 & 6.622 & 6.603 & 5.352 & 5.342 & 4.390 & 4.384 \\
\hline 3.00 & 8.055 & 8.019 & 6.514 & 6.495 & 5.372 & 5.761 \\
\hline
\end{tabular}

(e)

\begin{tabular}{|c|c|c|c|c|c|c|}
\hline \multirow[t]{2}{*}{$k$} & \multicolumn{2}{|c|}{$\mathrm{L}=13$} & \multicolumn{2}{|c|}{$\mathrm{L}=14$} & \multicolumn{2}{|c|}{$\mathrm{L}=15$} \\
\hline & Triplet & Singlet & Triplet & Singlet & Triplet & Singlet \\
\hline 0.91 & 0.576 & 0.576 & 0.466 & 0.466 & 0.381 & 0.381 \\
\hline 0.92 & 0.589 & 0.589 & 0.476 & 0.476 & 0.390 & 0.390 \\
\hline 0.95 & 0.626 & 0.626 & 0.506 & 0.506 & 0.414 & 0.414 \\
\hline 0.98 & 0.664 & 0.664 & 0.536 & 0.536 & 0.439 & 0.439 \\
\hline 1.00 & 0.691 & 0.691 & 0.558 & 0.558 & 0.457 & 0.457 \\
\hline 1,02 & 0.717 & 0.717 & 0.580 & 0.580 & 0.475 & 0.475 \\
\hline 1.05 & 0.756 & 0.756 & 0.611 & 0.611 & 0.500 & 0.500 \\
\hline 1.10 & 0.824 & 0.824 & 0.666 & 0.666 & 0.545 & 0.545 \\
\hline 1.20 & 0.968 & 0.968 & 0.782 & 0.782 & 0.640 & 0.640 \\
\hline 1.30 & 1.121 & 1.121 & 0.907 & 0.907 & 0.742 & 0.742 \\
\hline 1.40 & 1.298 & 1.298 & 1.053 & 1.053 & 0.865 & 0.865 \\
\hline 1.50 & 1.492 & 1.492 & 1.217 & 1.217 & 1.004 & 1.004 \\
\hline 1.60 & 1.677 & 1.677 & 1.370 & 1.370 & 1.133 & 1.133 \\
\hline 1.65 & 1.771 & 1.771 & 1.449 & 1.449 & 1.198 & 1.198 \\
\hline 1.69 & 1.846 & 1.846 & 1.511 & 1.511 & 1.251 & 1.251 \\
\hline 1.70 & 1.866 & 1.866 & 1.528 & 1.528 & 1.265 & 1.265 \\
\hline 1.71 & 1.885 & 1.885 & 1.544 & 1.544 & 1.278 & 1.278 \\
\hline 1.75 & 1.963 & 1.963 & 1.609 & 1.609 & 1.333 & 1.333 \\
\hline 1.80 & 2.058 & 2.058 & 1.688 & 1.688 & 1.400 & 1.400 \\
\hline
\end{tabular}


Table 3. Cont.

\begin{tabular}{llllll}
\hline 2.00 & 2.455 & 2.455 & 2.023 & 2.023 & 1.684 \\
2.10 & 2.652 & 2.652 & 2.192 & 2.191 & 1.827 \\
2.20 & 2.850 & 2.850 & 2.361 & 2.361 & 1.972 \\
2.30 & 3.049 & 3.049 & 2.531 & 2.530 & 2.119 \\
2.40 & 3.247 & 3.245 & 2.703 & 2.703 & 2.261 \\
2.50 & 3.445 & 3.443 & 2.874 & 2.873 & 2.416 \\
2.60 & 3.640 & 3.637 & 3.044 & 3.043 & 2.565 \\
3.00 & 4.493 & 4.487 & 3.797 & 3.794 & 3.236 \\
\hline
\end{tabular}

\section{Rate Coefficients}

In solar and astrophysics, parameters, namely the electron density and temperature, of the plasma are required. These parameters can be determined by solving the statistical equilibrium equations involving electron collision rate coefficients and radiative transition rates. The excitation rate coefficient is defined by

$$
C_{i j}(T)=\frac{8.63 \times 10^{-6}}{g_{i}(T)^{0.5}} \int_{E t h}^{\infty} \Omega_{i j} e^{-E_{i} / k T} d\left(E_{i} / k T\right)
$$

where $\Omega_{i j}=g_{i} E_{i} \sigma_{i j}$ is the collision strength at the incident energy $E_{i}, g_{i}=2$ is the weight factor for the initial state, $k$ is Boltzmann's constant, $\sigma_{i j}$ are the excitation cross-sections given in Table 1, and the lower limit in the integral is the threshold energy for excitation. The excitation rate coefficients for excitation to the $n S$ states, $n=2-6$, are given in Table 4 . Defining $E_{i j}=E_{j}-E_{i}$, it can be shown from the principle of detailed balance that the de-excitation rate coefficients are given by

$$
C_{j i}=\frac{g_{i}}{g_{j}} e^{E_{i j} / k T} C_{i j}
$$

Table 4. Excitation rate coefficients for $n S$ states, $n=2$ to 6 , at various temperatures.

\begin{tabular}{cccccc}
\hline T (K) & \multicolumn{5}{c}{ Excitation Rate Coefficients } \\
\hline & $2 S$ & $3 S$ & $4 S$ & $5 S$ & $6 S$ \\
\hline $2(+4)$ & $1.60(-11)$ & $5.79(-12)$ & $5.56(-12)$ & $1.87(-12)$ & $1.04(-12)$ \\
$5(+4)$ & $3.76(-11)$ & $3.67(-10)$ & $3.24(-10)$ & $1.27(-10)$ & $7.67(-11)$ \\
$1(+5)$ & $1.05(-9)$ & $1.16(-9)$ & $9.24(-10)$ & $4.01(-10)$ & $2.53(-10)$ \\
$2(+5)$ & $1.67(-9)$ & $1.45(-9)$ & $1.10(-9)$ & $5.16(-10)$ & $3.42(-10)$ \\
$5(+5)$ & $1.41(-9)$ & $8.93(-10)$ & $6.67(-10)$ & $3.30(-10)$ & $2.30(-10)$ \\
$1(+6)$ & $7.94(-9)$ & $4.43(-10)$ & $3.31(-10)$ & $1.67(-10)$ & $1.19(-10)$ \\
$2(+6)$ & $3.58(-10)$ & $1.88(-10)$ & $1.40(-10)$ & $7.16(-11)$ & $5.16(-11)$ \\
$5(+6)$ & $1.05(-10)$ & $5.31(-11)$ & $3.98(-11)$ & $2.04(-11)$ & $1.48(-11)$ \\
$1(+7)$ & $3.91(-11)$ & $1.95(-11)$ & $1.46(-11)$ & $7.53(-12)$ & $5.48(-12)$ \\
\hline
\end{tabular}

\section{Comparison with Positron Impact Excitation}

As it was mentioned in the introduction that positrons are as important as electrons to describe the opacity of the Sun and interstellar space, it is worth having a comparison of the excitation cross-sections by electron and positron impact on hydrogen atoms. A comparison with the positron impact excitation [3] is given in Table 5. Positron impact cross-sections are higher than the electron impact cross sections. 
Table 5. Comparison of the positron and electron impact excitation of the sum of the $n=2$ to 6 states of the atomic hydrogen.

\begin{tabular}{ccc}
\hline Incident $\boldsymbol{k}$ & Positron Impact & Electron Impact \\
\hline 0.87 & $9.35(-2)$ & $6.82(-2)$ \\
0.88 & $1.69(-1)$ & $1.12(-1)$ \\
0.89 & $2.14(-1)$ & $1.30(-1)$ \\
0.90 & $2.46(-1)$ & $1.36(-1)$ \\
0.92 & $2.90(-1)$ & $1.35(-1)$ \\
0.95 & $3.28(-1)$ & $1.62(-1)$ \\
1.00 & $3.79(-1)$ & $4.00(-1)$ \\
1.10 & $4.57(-1)$ & $4.72(-1)$ \\
1.20 & $3.71(-1)$ & $5.23(-1)$ \\
1.40 & $3.13(-1)$ & $3.18(-1)$ \\
1.50 & $2.86(-1)$ & $2.32(-1)$ \\
1.60 & $2.63(-1)$ & $2.04(-1)$ \\
1.80 & $2.24(-1)$ & $1.34(-1)$ \\
2.00 & $1.90(-1)$ & $1.03(-1)$ \\
2.50 & $1.25(-1)$ & $7.12(-2)$ \\
\hline
\end{tabular}

\section{Threshold Law}

Wigner [24] has emphasized the importance of long-range forces in the threshold region. The long-range forces were included in the present calculation. It has been shown by Sadeghpour et al. [25] that in the threshold region the cross sections are inversely proportional to $\left(\ln k_{f}\right)^{2}$. The cross-sections calculated here near the threshold region can be fitted to $-0.44844+\frac{27.969115}{\left(\ln k_{f}\right)^{2}}-\frac{382.92}{\left(\ln k_{f}\right)^{3}}$. The fit is shown in Figure 6 and the solid line goes through all the calculated points. Threshold behavior can be a useful diagnostic of long-range potentials [24].

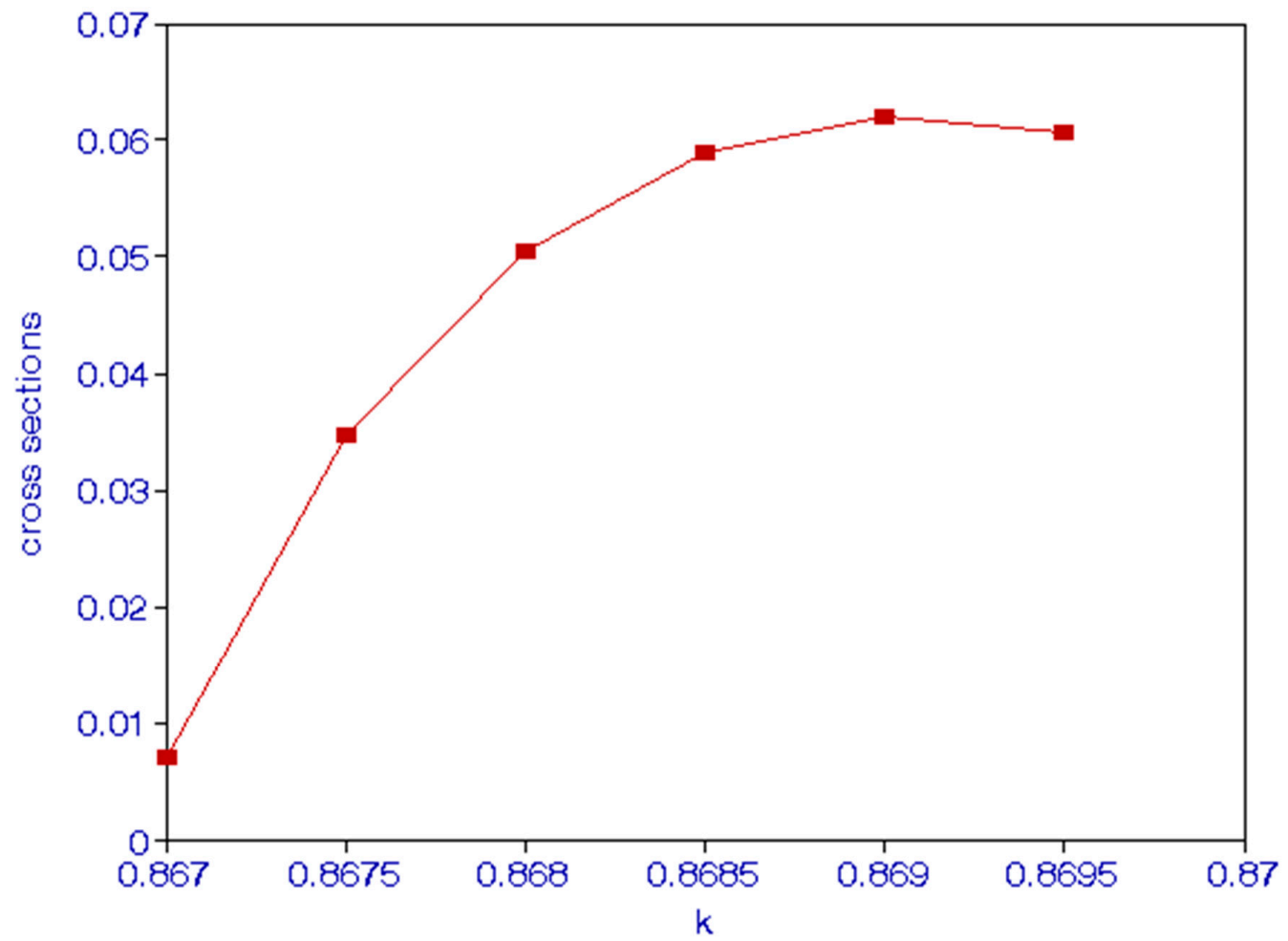

Figure 6. (Color online) $2 S$ excitation cross sections $\left(\pi a_{0}^{2}\right)$ as a function of the incident $k$ (Ryd) in the threshold region, the solid line represents the fit to the squares (calculated cross sections). 


\section{Conclusions}

Calculations were carried for excitation from $1 S$ state to $n S$ states of the hydrogen atom, $n=2-6$, by electron impact, in the distorted-wave approximation using the variational polarized-orbital method [10]. An approximation is called a distorted-wave approximation when the channel coupling between the initial and final states is neglected, as in the present calculation. Cross-sections have been calculated from $k=0.87$ to 3.0. Present results have been compared to those obtained using other approaches. Rate coefficients for excitation, required for diagnostic purposes, are calculated using the excitation crosssections. A comparison of the electron impact excitation cross sections with the positron impact excitation cross sections was made because positrons are found to be important in solar physics [26]. The cross-sections in the threshold region are shown to be proportional to $\left(\ln k_{f}\right)^{-2}$, as shown in Figure 6. The calculations were carried out in the quadruple precision. Therefore, results obtained are expected to be accurate.

Funding: The research received no external funding.

Acknowledgments: Thanks are extended to Aaron Temkin for helpful comments and critical reading of the manuscript.

Conflicts of Interest: The author declares no conflict of interest.

\section{Appendix A}

The hydrogenic functions are given below:

$$
\begin{gathered}
\phi_{n s}(\vec{r})=R_{n s}(r) /(4 \pi)^{0.5} \\
R_{1 s}(r)=2 e^{-r} \\
R_{2 s}(r)=\frac{(2-r)}{(8)^{0.5}} e^{-r / 2} \\
R_{3 s}(r)=\frac{2}{(27)^{0.5}}\left(1-\frac{2 r}{3}-\frac{2 r^{2}}{27}\right) e^{-r / 3} \\
R_{4 s}(r)=\frac{1}{96}\left(24-18 r+3 r^{2}-\frac{r^{3}}{8}\right) e^{-r / 4} \\
R_{5 s}(r)=\frac{1}{300(5)^{0.5}}\left(120-96 r+19.2 r^{2}-1.28 r^{3}+0.0256 r^{4}\right) e^{-r / 5} \\
R_{6 s}(r)=\frac{1}{2160(6)^{0.5}}\left(720-600 r+400 r^{2}-\frac{100}{9} r^{3}+\frac{10}{27} r^{4}-\frac{r^{5}}{243}\right) e^{-r / 6}
\end{gathered}
$$

\section{References}

1. Leventhal, M. Recent Balloon Observations of the Galactic Center $511 \mathrm{keV}$ annihilation line. Adv. Space Res. 1991, 11, 157. [CrossRef]

2. Bhatia, A.K. Excitation of the $2 S$ State of Atomic Hydrogen by Electron Impact. Atoms 2018, 6, 7. [CrossRef]

3. Bhatia, A.K. Positron Impact Excitation of the $n S$ States of Atomic Hydrogen. Atoms 2020, 8, 9. [CrossRef]

4. Burke, P.G.; Schey, H.M.; Smith, K. Collisions of Slow Electrons and Positrons with Atomic Hydrogen. Phys. Rev. 1963, 120, 1258. [CrossRef]

5. Rudge, M.R.H. The scattering of electrons by hydrogen atoms. Proc. Phys. Soc. 1965, 86, 765-772. [CrossRef]

6. Bartlett, P.L.; Stelbovics, A.T. Complete direct method for electron-hydrogen scattering. Applications to the collinear and Temkin models. Phys. Rev. A 2004, 69, 022703. [CrossRef]

7. Jones, S.; Stelbovics, A.T. Complete Numerical Solutions of Electron-Hydrogen Model Collision Problem above the ionization Threshold. Phys. Rev. Lett. 2000, 84, 1878. [CrossRef]

8. Callaway, J. Scattering of electrons by atomic hydrogen at intermediate energies: Elastic scattering and $n=2$ excitation from 12 to $54 \mathrm{eV}$. Phys. Rev. A 1985, 32, 775-783. [CrossRef] 
9. Scott, M.P.; Schulz, T.T.; Walters, H.R.; Burke, P.G. Electron scattering by atomic hydrogen at intermediate energies: Integrated elastic, is-2s and 1s-2p cross sections. J. Phys. B 1989, 22, 3055-3077. [CrossRef]

10. Bhatia, A.K. Hybrid theory of electron-hydrogen elastic scattering. Phys. Rev. A 2007, 75, 032713. [CrossRef]

11. Bhatia, A.K. Applications of P-wave hybrid theory to the scattering of electrons from $\mathrm{He}^{+}$and resonances in $\mathrm{He}^{2}$ and $\mathrm{H}^{-}$. Phys. Rev. A 2012, 86, 032709. [CrossRef]

12. Bhatia, A.K. Photoionization in two-electron systems. Phys. Rev. A 2013, 87, 042705. [CrossRef]

13. Calhoun, R.V.; Madison, D.H.; Sheldon, W.N. Excitation of the $n=2$ state of atomic hydrogen by electron impact in the distorted-wave approximation. Phys. Rev. A 1976, 14, 1380-1387. [CrossRef]

14. Temkin, A.; Lamkin, J.C. Application of the Method of Polarized Orbitals to the Scattering of Electrons from Hydrogen. Phys. Rev. 1961, 121, 788-794. [CrossRef]

15. Shertzer, J.; Temkin, A. Direct calculation of the scattering amplitude without partial-wave analysis. III. Inclusion of correlation effects. Phys. Rev. A 2006, 74, 052701. [CrossRef]

16. Omidvar, K. 2s and 2p Electron-Impact Excitation in Atomic Hydrogen. Phys. Rev. 1964, 133, A970. [CrossRef]

17. Kauppila, W.E.; Ott, W.R.; Fite, W.L. Excitation of Atomic Hydrogen to the Metastable ${ }^{2} S_{1 / 2}$ State by Electron-Impact. Phys. Rev. A 1970, 1, 1099-1108. [CrossRef]

18. Callaway, J.; Unikrishnan, K.; Oza, D.H. Optical potential of electron-hydrogen scattering at intermediate energies. Phys. Rev. A 1987, 36, 2576. [CrossRef]

19. Whelan, C.T.; McDowell, M.R.C.; Edmunds, P.W. Electron impact excitation of atomic hydrogen. J. Phys. B 1987, $20,1587$. [CrossRef]

20. Mahan, A.H.; Gallagher, A.; Smith, S.J. Electron impact excitation of the 3S, 3P, and 3D states of H. Phys. Rev. A 1976, 13,156 [CrossRef]

21. Bartlett, P.L.; Williams, J.F.; Bray, I.; Mikoza, A.G.; Stelbovics, A.T. Differential and integrated cross sections for excitation to the 3s, $3 p$, and 3d states of atomic hydrogen by electron impact below the $n=4$ threshold. Phys. Rev. A 2006, 74, 022714. [CrossRef]

22. Bray, I.; Stelbovics, A.T. Convergent close-coupling calculations of electron-hydrogen scattering. Phys. Rev. A 1992, 46, 6995 [CrossRef] [PubMed]

23. Poet, R. The exact solution for a simplified model of electrons scattering by hydrogen atoms. J. Phys. B 1978, 11, 3081-3094. [CrossRef]

24. Wigner, E.P. On the behavior of cross sections near threshold. Phys. Rev. 1948, 73, 1002. [CrossRef]

25. Sadeghpour, H.R.; Cavagnero, M.J.; Esry, B.D.; Fabrikant, I.T.; Macek, J.H.; Rau, A.R.P. Collisions near threshold in atomic and molecular physics. J. Phys. B 2000, 33, R93-R140. [CrossRef]

26. Gopalswamy, N. Positron Processes in the Sun. Atoms 2020, 8, 14. [CrossRef] 